\title{
RANDOM WALKS ON DISCRETE ABELIAN GROUPS
}

\section{Margaryta Myronyuk}

\begin{abstract}
In the present paper we find necessary and sufficient conditions for recurrence of random walks on arbitrary subgroups of the group of rational numbers $\mathbb{Q}$.
\end{abstract}

2010 Mathematics Subject Classification: Primary 60G50; Secondary 60B15, 43A25.

Key words and phrases: random walk, Abelian group.

\section{Introduction}

Let $(\Omega, \mathfrak{A}, P)$ be a probability space, $X$ be a countable discrete Abelian groups, $\mu$ be a distribution on $X$. Recall that a random walk on a group $X$ generated by the distribution $\mu$ is a sequence

$$
S_{n}=\xi_{1}+\ldots+\xi_{n}, \quad n=1,2, \ldots,
$$

where $\xi_{j}$ are independent identically distributed with the distribution $\mu$ random variables defined on $(\Omega, \mathfrak{A}, P)$ with values on $X$. The random walk on the group $X$ is said to be recurrent if all elements of $X$ are recurrent i.e. for every $x \in X$ the equality

$$
P\left\{\omega \in \Omega: S_{n}(\omega)=x \text { for infinitely many indices } n \in \mathbf{N}\right\}=1
$$

holds.

Denote by $\mathbb{Z}$ the additive group of integers, by $\mathbb{Q}$ the additive group of rational numbers considering in the discrete topology, and by $\mathbb{Z}(k)$ the finite cyclic group. R. M. Dudley ( [2]) proved that there exists a recurrent random walk on a countable Abelian group $X$ iff $X$ contains no subgroup isomorphic to $\mathbb{Z}^{3}$. Dudley's proof is not constructive and therefore it is natural to look for other effective recurrence conditions.

Such conditions on $\mu$ were studied

(a) on the weak direct product $\mathbb{Z}(2)^{\aleph_{0} *}([1])$,

(b) on the weak direct product $\mathbf{P}_{i \in \mathbb{N}}^{*} \mathbb{Z}\left(k_{i}\right)$ ( [4]),

(c) on the factor group $\mathbb{Q} / \mathbb{Z}$ and its subgroups ( [4]),

(d) on the weak direct product $\mathbb{Z}(k)^{\aleph_{0} *}([5])$,

(e) on subgroups $H_{p}=\left\{\frac{m}{p^{n}}: n=1,2, \ldots ; m \in \mathbb{Z}\right\}$ of the group $\mathbb{Q}([5])$,

(f) on groups of the form $\mathbb{Z}^{k} \times \mathbf{P}_{i \in \mathbb{N}}^{*} \mathbb{Z}\left(k_{i}\right)$ ( [7]).

In the present paper we find necessary and sufficient conditions for recurrence of random walks on arbitrary subgroups of the group of rational numbers $\mathbb{Q}$.

Let $X$ be a locally compact second countable Abelian group, $Y=X^{*}$ be its character group, and $(x, y)$ be the value of a character $y \in Y$ at an element $x \in X$. Let

$$
\widehat{\mu}(y)=\int_{X}(x, y) d \mu(x)
$$


be the characteristic function of a distribution $\mu$ on $X$. Denote by $m_{X}$ the Haar measure on $X$.

The following recurrence criterion was proved in [8].

Theorem A ( [8]). Let $X$ be a countable discrete Abelian group. Let $\mu$ be a distribution on $X$. A random walk defined by the distribution $\mu$ is recurrent iff

$$
\int_{Y} R e \frac{1}{1-\hat{\mu}(y)} d m_{Y}=\infty
$$

In cases (b), (c), (d), (f) authors of corresponding papers use Theorem A to obtain necessary and sufficient conditions for recurrence of random walks. In these cases the character groups have quite simple structure.

The case (e) is more complicated. Authors of [5] state that Theorem A is useless for obtaining necessary and sufficient conditions for recurrence of random walks on a group $X$ if its character

group $Y$ has a complicated structure as, for example, the group $H_{p}=\left\{\frac{m}{p^{n}}: n=1,2, \ldots ; m \in \mathbb{Z}\right\}$. In this case the character group $Y$ is a $p$-adic solenoid. In [5] necessary and sufficient conditions for recurrence of random walks on a group $H_{p}$ were obtained without using of Theorem $\mathrm{A}$.

In the present paper we do use Theorem A and find necessary and sufficient conditions for recurrence of random walks on arbitrary subgroups of the group of rational numbers $\mathbb{Q}$. In this case the character group $Y$ is a $a$-adic solenoid. Note that the results of the paper [5] for the groups $H_{p}$ follows directly from our paper.

\section{Notation and definitions}

In the present paper we use some results from the Pontryagin duality theory (see [6]).

In the paper we consider random walks on arbitrary subgroups of the group of rational numbers not isomorphic to $\mathbb{Z}$.

Let $a=\left(a_{1}, a_{2}, \ldots\right)$ be a sequence of integers where all $a_{j}>1$. Consider a group

$$
H_{a}=\left\{\frac{m}{a_{1} a_{2} \ldots a_{n}}: n=1,2, \ldots ; m \in \mathbb{Z}\right\} .
$$

It is well-known that any subgroup of the group $\mathbb{Q}$ not isomorphic to $\mathbb{Z}$ has the form (3) for some $a=\left(a_{1}, a_{2}, \ldots\right)$. Particularly, if all $a_{j}=p$ then we obtain the group of $H_{p}=\left\{\frac{m}{p^{n}}: n=1,2, \ldots ; m \in \mathbb{Z}\right\}$.

In order to apply Theorem $\mathrm{A}$, we have to describe the character group of the group $H_{a}$.

Let $\Delta_{a}$ be a group of $a$-adic integers. Consider the group $\mathbb{R} \times \Delta_{a}$. Let $B$ the subgroup of $\mathbb{R} \times \Delta_{a}$ of the form $B=\left\{(n, n \mathbf{u}\}_{n=-\infty}^{\infty}\right.$, where $\mathbf{u}=(1,0,0, \ldots, 0, \ldots) \in \Delta_{a}$. The factor group $\Sigma_{a}=\mathbb{R} \times \Delta_{a} / B$ is called the $a$-adic solenoid. The group $\Sigma_{a}$ is compact and connected (see [6, $\S 10]$ ). The group $\Sigma_{a}$ is topologically isomorphic to the character group of the group $H_{a}$ (see [6, $(25.3)])$.

Denote by $\mathbb{T}$ the circle group (the one-dimensional torus), i.e. $\mathbb{T}=\{z \in \mathbb{C}:|z|=1\}$. It is convenient for us to use another consideration of the $a$-adic solenoid as a subgroup of the infinite-dimensional torus $\mathbb{T}^{\aleph_{0}}$.

Consider the mapping $f: \mathbb{R} \times \Delta_{a} \longrightarrow \mathbb{T}^{\aleph_{0}}$, defined by 


$$
f(t, \mathbf{y}) \longmapsto z=\left(z_{1}, z_{2}, \ldots\right), z_{j}=\exp \left(\frac{2 \pi i}{a_{1} \cdots a_{j}}\left(t-\left(y_{0}+a_{1} y_{1}+\ldots+a_{1} a_{2} \ldots a_{j-1} y_{j-1}\right)\right)\right)
$$

where $t \in \mathbb{R}, \mathbf{y}=\left(y_{0}, y_{1}, \ldots\right) \in \Delta_{a}$.

It is not difficult to verify that $f$ is a continuous homomorphism, $\operatorname{Ker} f=B$ and $G=\operatorname{Im} f=\left\{z=\left(z_{1}, z_{2}, \ldots, z_{n}, \ldots\right) \in \mathbb{T}^{\aleph_{0}}: z_{k}^{a_{k}}=z_{k-1}\right\}$ is a closed subgroup of the infinitedimensional torus $\mathbb{T}^{\aleph_{0}}$. Then $G \cong \Sigma_{a}$.

The consideration of the $a$-adic solenoid $\Sigma_{a}$ as the subgroup

$$
G=\left\{z=\left(z_{1}, z_{2}, \ldots, z_{n}, \ldots\right) \in \mathbb{T}^{\aleph_{0}}: z_{k}^{a_{k}}=z_{k-1}\right\}
$$

allows us to verify easily the following: if $h=\frac{m}{a_{1} a_{2} \ldots a_{n}}$ is a character of the group $\Sigma_{a}$, then $(z, h)=z_{n+1}^{m}, z=\left(z_{1}, z_{2}, \ldots, z_{n}, \ldots\right) \in G$.

\section{Main results}

Let $a=\left(a_{1}, a_{2}, \ldots\right)$ be a sequence of integers where all $a_{j}>1$. Consider the group $X=H_{a}$. Note that numbers

$$
e_{ \pm 0}= \pm 1, \quad e_{ \pm 1}= \pm \frac{1}{a_{1}}, \quad e_{ \pm 2} \pm \frac{1}{a_{1} a_{2}}, \quad \ldots, \quad e_{ \pm n}= \pm \frac{1}{a_{1} \ldots a_{n}}, \quad \ldots
$$

are natural generators of the group $X$.

We consider on $X$ a distribution $\mu$ of the form

$$
\mu\left\{e_{ \pm j}\right\}=\frac{q_{j}}{2}, \quad \sum_{j=0}^{\infty} q_{j}=1, \quad q_{j} \geq 0 .
$$

The distribution $\mu$ defines a random walk on $X$.

For a compact group $Y$ we suppose that a Haar measure $m_{Y}$ is normalized in such a way that $m_{Y}(Y)=1$.

In the following theorem we obtain sufficient conditions for recurrence of a random walk defined by a distribution $\mu$ on the group $X=H_{a}$.

Theorem 1. Let $X=H_{a}$, where $a=\left(a_{1}, a_{2}, \ldots\right), a_{j}>1$. Let $\mu$ be a distribution on $X$ of the form (4). Consider on $X$ a random walk defined by $\mu$. The condition

$$
\sum_{n=1}^{\infty} \frac{1}{a_{1} \ldots a_{n} \sqrt{q_{n}+q_{n+1}+\ldots}}=\infty
$$

is sufficient for recurrence of a random walk defined by $\mu$ on $X$.

Proof. The proof of Theorem 1 is based on Theorem A. The character group of the group $X$ is topologically isomorphic to the group $\Sigma_{a}$. In order not to complicate the notation, we will assume that $Y=\Sigma_{a}$. It is convenient to consider the realization of the $a$-adic solenoid as a subgroup in $\mathbb{T}^{\aleph_{0}}$. Then each element of $Y$ is a sequence $y=\left(y_{1}, y_{2}, \ldots\right)$, where $y_{n} \in \mathbb{T}$, $y_{n}^{a_{n}}=y_{n-1}$. Put $y_{n}=e^{i b_{n}}$. Thus the sequence $\left(y_{1}, y_{2}, \ldots\right)$ corresponds to a sequence $\left(b_{1}, b_{2}, \ldots\right)$. 

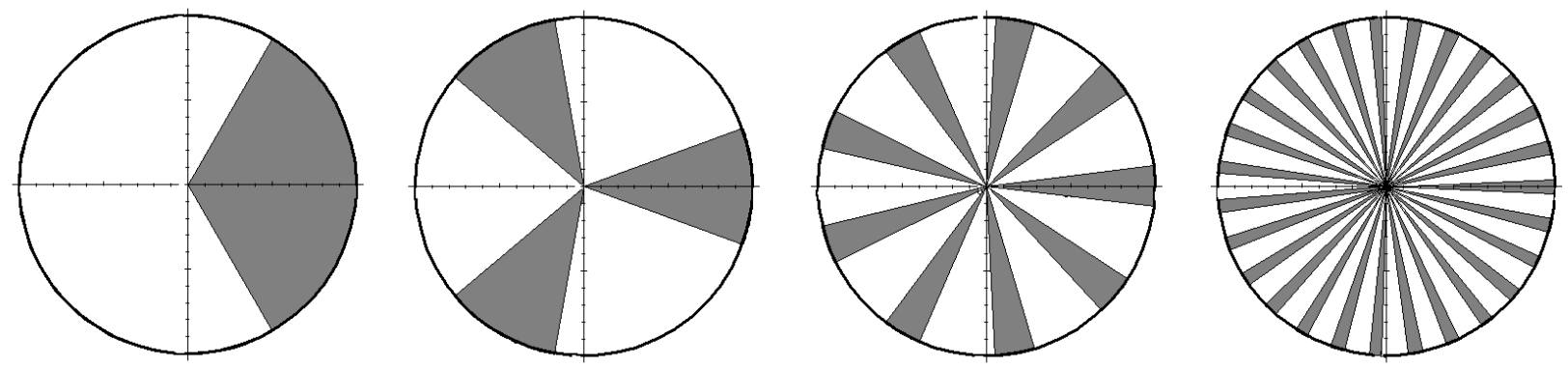

Figure 1: The domains of variation of elements $b_{1}, b_{2}, b_{3}, b_{4}$ respectively in the subset $Y \backslash E_{0}$ for the case when each $a_{j}=3$.

Numbers $b_{n}$ are defined modulo $2 \pi$. We will take these numbers either in the interval $[0,2 \pi)$ or in the interval $[-\pi, \pi)$ depending on how it is convenient for us. Regardless of an interval in which we take numbers $b_{n}$, misunderstandings will not arise. Note that

$$
a_{n+1} b_{n+1}=b_{n}(\bmod 2 \pi) .
$$

Note also that $\left(e_{ \pm j}, y\right)=e^{ \pm i b_{j+1}}=\cos b_{j+1} \pm i \sin b_{j+1}$. Then

$$
\hat{\mu}(y)=\int_{X}(x, y) d \mu(x)=\frac{1}{2} \sum_{j=0}^{\infty} q_{j}\left(e_{j}, y\right)+\frac{1}{2} \sum_{j=0}^{\infty} q_{j}\left(e_{-j}, y\right)=\sum_{j=0}^{\infty} q_{j} \cos b_{j+1} .
$$

We will build by induction on the group $Y$ a system of non overlapping sets $E_{0}, E_{1}, \ldots, E_{n}, \ldots$ such that

$$
\sum_{n=0}^{\infty} m_{Y}\left(E_{n}\right)=1
$$

Put

$$
E_{0}=\left\{y \in Y: b_{1} \notin\left[-\frac{\pi}{a_{1}}, \frac{\pi}{a_{1}}\right]\right\} .
$$

Using the invariance of the Haar measure we obtain that

$$
m_{Y}\left(E_{0}\right)=\frac{a_{1}-1}{a_{1}}
$$

Note

$$
\begin{gathered}
Y \backslash E_{0}=\left\{y \in Y: b_{1} \in\left[-\frac{\pi}{a_{1}}, \frac{\pi}{a_{1}}\right], b_{2} \in\left[\frac{2 \pi k}{a_{2}}-\frac{\pi}{a_{1} a_{2}}, \frac{2 \pi k}{a_{2}}+\frac{\pi}{a_{1} a_{2}}\right]\left(k=0,1, \ldots, a_{2}-1\right), \ldots\right. \\
\left.\ldots, b_{n} \in\left[\frac{2 \pi k}{a_{n}}-\frac{\pi}{a_{1} \ldots a_{n}}, \frac{2 \pi k}{a_{n}}+\frac{\pi}{a_{1} \ldots a_{n}}\right]\left(k=0,1, \ldots, a_{n}-1\right), \ldots\right\}
\end{gathered}
$$

See Figure 1 for the case when each $a_{j}=3$.

$$
\begin{gathered}
\text { Put } \\
\qquad E_{1}=\left\{y \in Y \backslash E_{0}: b_{2} \notin\left[-\frac{\pi}{a_{1} a_{2}}, \frac{\pi}{a_{1} a_{2}}\right]\right\}= \\
\left\{y \in Y \backslash E_{0}: b_{1} \in\left[-\frac{\pi}{a_{1}}, \frac{\pi}{a_{1}}\right], b_{2} \in\left[\frac{2 \pi k}{a_{2}}-\frac{\pi}{a_{1} a_{2}}, \frac{2 \pi k}{a_{2}}+\frac{\pi}{a_{1} a_{2}}\right]\left(k=1, \ldots, a_{2}-1\right)\right\} .
\end{gathered}
$$



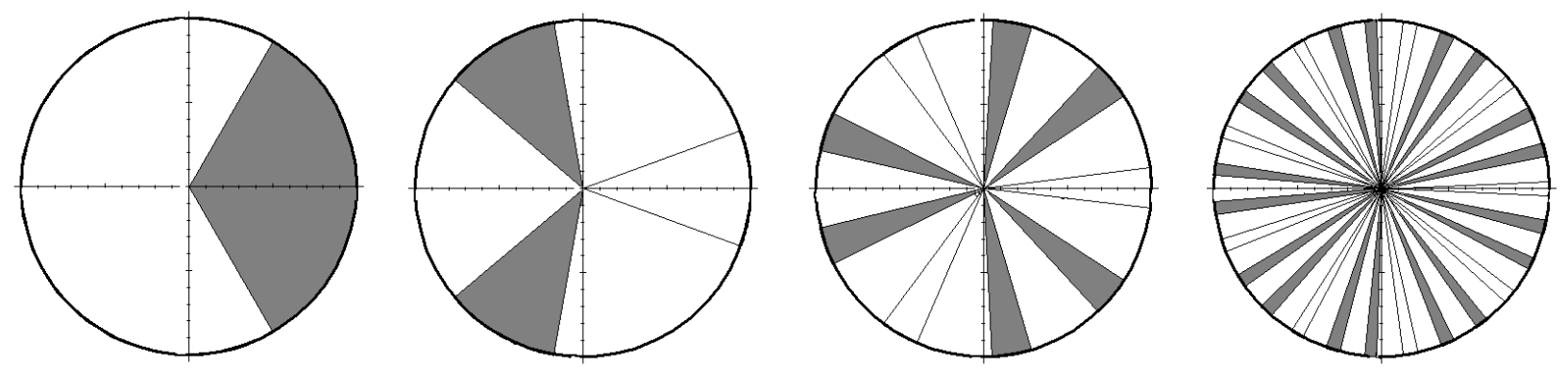

Figure 2: The domains of variation of elements $b_{1}, b_{2}, b_{3}, b_{4}$ respectively in the subset $E_{1}$ for the case when each $a_{j}=3$.

It is easy to see that $m_{Y}\left(E_{1}\right)=\frac{a_{2}-1}{a_{1} a_{2}}$ (see Figure 2 for the case when each $a_{j}=3$ ).

We define by induction a sequence of sets

$$
E_{n}=\left\{y \in Y \backslash \bigcup_{j=0}^{n-1} E_{j}: b_{n+1} \notin\left[-\frac{\pi}{a_{1} \ldots a_{n+1}}, \frac{\pi}{a_{1} \ldots a_{n+1}}\right]\right\} .
$$

Actually sets $E_{n}$ can be defined by means of the coordinate $b_{n+1}$. For a better understanding we note that

$$
\begin{gathered}
E_{n}=\left\{y \in Y: b_{n+1} \in\left[\frac{2 \pi k}{a_{n+1}}-\frac{\pi}{a_{1} a_{2} \ldots a_{n+1}}, \frac{2 \pi k}{a_{n+1}}+\frac{\pi}{a_{1} a_{2} \ldots a_{n+1}}\right]\left(k=1, \ldots, a_{n+1}-1\right)\right\}= \\
=\left\{y \in Y \backslash \bigcup_{j=0}^{n-1} E_{j}: b_{1} \in\left[-\frac{\pi}{a_{1}}, \frac{\pi}{a_{1}}\right], b_{2} \in\left[-\frac{\pi}{a_{1} a_{2}}, \frac{\pi}{a_{1} a_{2}}\right], \ldots, b_{n} \in\left[-\frac{\pi}{a_{1} a_{2} \ldots a_{n}}, \frac{\pi}{a_{1} a_{2} \ldots a_{n}}\right],\right. \\
\left.b_{n+1} \in\left[\frac{2 \pi k}{a_{n+1}}-\frac{\pi}{a_{1} a_{2} \ldots a_{n+1}}, \frac{2 \pi k}{a_{n+1}}+\frac{\pi}{a_{1} a_{2} \ldots a_{n+1}}\right]\left(k=1, \ldots, a_{n+1}-1\right)\right\} .
\end{gathered}
$$

See Figure 3 for the case when each $a_{j}=3$.

Using (11) and invariance of the Haar measure it is easy to verify by induction that

$$
m_{Y}\left(E_{n}\right)=\frac{a_{n+1}-1}{a_{1} \ldots a_{n+1}}
$$

for all $n=0,1,2, \ldots$.

By construction, we have obtained that $E_{i} \cap E_{j}=\emptyset$ for $i \neq j$. We have

$\sum_{n=0}^{\infty} m_{Y}\left(E_{n}\right)=\frac{a_{1}-1}{a_{1}}+\frac{a_{2}-1}{a_{1} a_{2}}+\frac{a_{3}-1}{a_{1} a_{2} a_{3}}+\ldots=1-\frac{1}{a_{1}}+\frac{1}{a_{1}}-\frac{1}{a_{1} a_{2}}+\frac{1}{a_{1} a_{2}}-\frac{1}{a_{1} a_{2} a_{3}}+\ldots=1$.

Let $\alpha_{n}$ be a number such that $0<\alpha_{n}<1$.

Put

$$
A_{n}=\left\{y \in E_{n}: b_{1} \in\left(-\frac{\pi \alpha_{n}}{a_{1}}, \frac{\pi \alpha_{n}}{a_{1}}\right)\right\}=\left\{y \in E_{n}: b_{2} \in\left(-\frac{\pi \alpha_{n}}{a_{1} a_{2}}, \frac{\pi \alpha_{n}}{a_{1} a_{2}}\right)\right\}=\ldots
$$



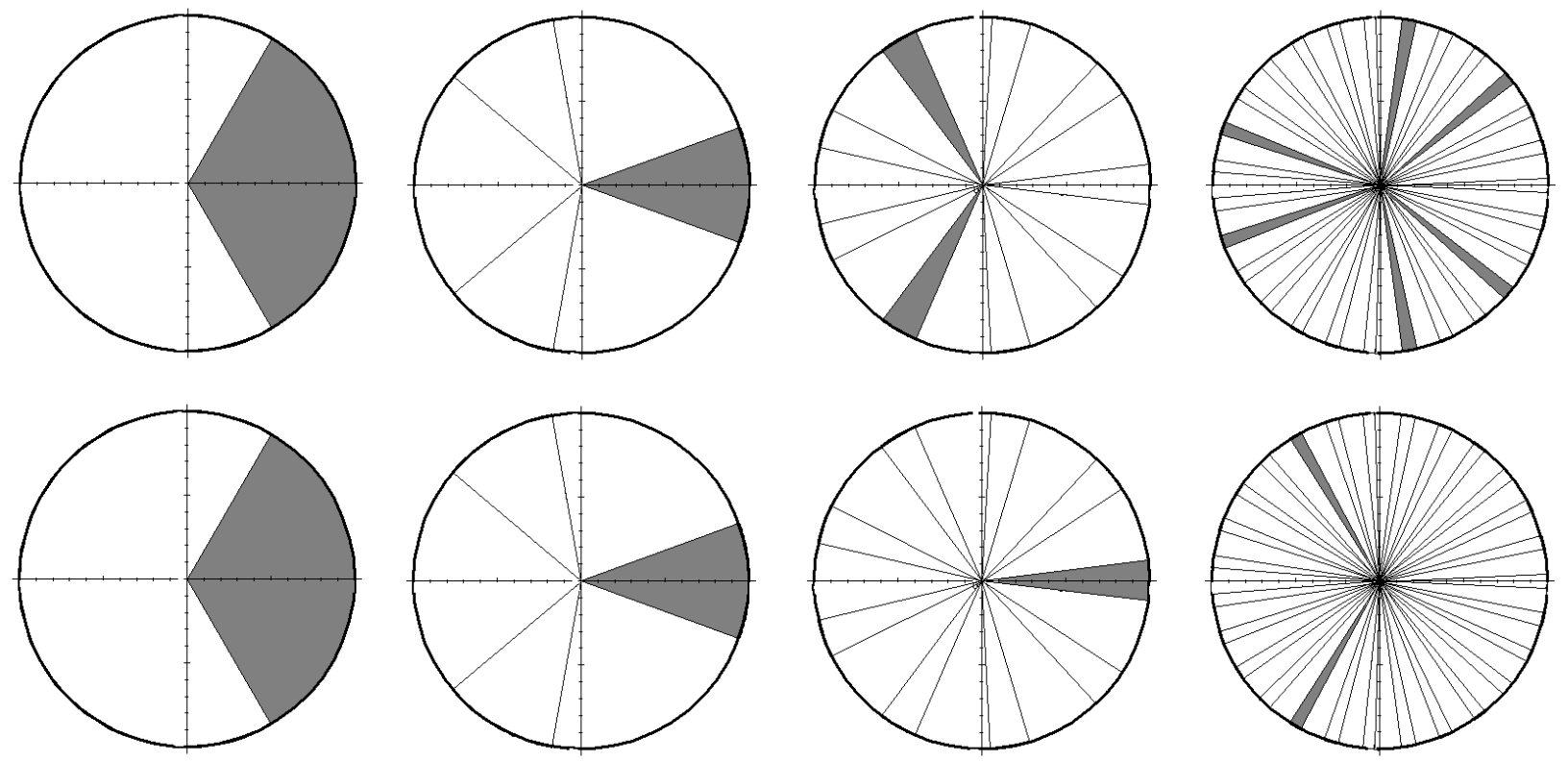

Figure 3: The domains of variation of elements $b_{1}, b_{2}, b_{3}, b_{4}$ respectively in the subsets $E_{2}$ and $E_{3}$ for the case when each $a_{j}=3$.

$$
=\left\{y \in E_{n}: b_{k} \in\left(-\frac{\pi \alpha_{n}}{a_{1} a_{2} \ldots a_{k}}, \frac{\pi \alpha_{n}}{a_{1} a_{2} \ldots a_{k}}\right)\right\}=\ldots
$$

Since the measure of $E_{n}$ coincides with the measure of projections on the $(n+1)$ circle, it is obvious that

$$
m_{Y}\left(A_{n}\right)=\alpha_{n} m_{Y}\left(E_{n}\right)=\alpha_{n} \frac{a_{n+1}-1}{a_{1} \ldots a_{n+1}} .
$$

We evaluate from below the sum of the following series

$$
\sum_{n=0}^{\infty} \int_{E_{n}} \frac{1}{1-\hat{\mu}(y)} d m_{Y}(y)
$$

We have

$$
\sum_{n=0}^{\infty} \int_{E_{n}} \frac{1}{1-\hat{\mu}(y)} d m_{Y}(y) \geq \sum_{n=1}^{\infty} \int_{A_{n}} \frac{1}{1-\hat{\mu}(y)} d m_{Y}(y) .
$$

We evaluate from above $1-\hat{\mu}(y)$ for $y \in A_{n}$. We have

$$
\begin{gathered}
1-\hat{\mu}(y)=1-\sum_{j=0}^{\infty} q_{j} \cos b_{j+1}=\sum_{j=0}^{\infty} q_{j}\left(1-\cos b_{j+1}\right) \leq \\
\leq q_{0}\left(1-\cos \frac{\pi \alpha_{n}}{a_{1}}\right)+q_{1}\left(1-\cos \frac{\pi \alpha_{n}}{a_{1} a_{2}}\right)+\ldots+q_{n-1}\left(1-\cos \frac{\pi \alpha_{n}}{a_{1} \ldots a_{n}}\right)+2\left(q_{n}+q_{n+1}+\ldots\right)
\end{gathered}
$$

Since

$$
\frac{2 t^{2}}{\pi^{2}} \leq 1-\cos t \leq \frac{t^{2}}{2}, \quad t \in\left[0, \frac{\pi}{2}\right]
$$


we can continue evaluation (17) in the following way.

$$
\begin{gathered}
1-\hat{\mu}(y) \leq \frac{\pi^{2} \alpha_{n}^{2}}{a_{1}^{2}}\left(1+\frac{1}{a_{2}^{2}}+\frac{1}{a_{2}^{2} a_{3}^{2}}+\ldots+\frac{1}{a_{2}^{2} \ldots a_{n}^{2}}\right)+2\left(q_{n}+q_{n+1}+\ldots\right) \\
\leq C_{1}\left(\alpha_{n}^{2}+q_{n}+q_{n+1}+\ldots\right),
\end{gathered}
$$

where $C_{1}$ is a constant which does not depend on $n$.

Taking into account (19) and (14), we can continue evaluation (16):

$$
\begin{aligned}
\sum_{n=0}^{\infty} \int_{E_{n}} \frac{1}{1-\hat{\mu}(y)} d m_{Y}(y) \geq \sum_{n=1}^{\infty} \int_{A_{n}} \frac{1}{1-\hat{\mu}(y)} d m_{Y}(y) \geq C_{2} \sum_{n=0}^{\infty} \frac{\alpha_{n}\left(a_{n+1}-1\right)}{a_{1} \ldots a_{n+1}\left(\alpha_{n}^{2}+q_{n}+q_{n+1}+\ldots\right)} \\
\geq C_{3} \sum_{n=0}^{\infty} \frac{\alpha_{n}}{a_{1} \ldots a_{n}\left(\alpha_{n}^{2}+q_{n}+q_{n+1}+\ldots\right)}
\end{aligned}
$$

where $C_{2}, C_{3}$ are constants which do not depend on $n$.

Note that for $a \in(0,1)$ the function $f(x)=\frac{x}{x^{2}+a}$ has a maximum value on $[0,1]$ at $x=\sqrt{a}$. Taking this into account from (20) we find that

$$
\sum_{n=0}^{\infty} \int_{E_{n}} \frac{1}{1-\hat{\mu}(y)} d m_{Y}(y) \geq C_{4} \sum_{n=0}^{\infty} \frac{1}{a_{1} \ldots a_{n} \sqrt{q_{n}+q_{n+1}+\ldots}}
$$

where $C_{4}$ is a constant which does not depend on $n$.

Now we can prove that condition (5) is sufficient for recurrence of a random walk defined by the distribution $\mu$. Indeed, suppose that condition (5) is satisfied and a random walk defined by the distribution $\mu$ is transient. Then Theorem A implies

$$
\int_{Y} \frac{1}{1-\hat{\mu}(y)} d m(y)<\infty .
$$

But then

$$
\int_{Y} \frac{1}{1-\hat{\mu}(y)} d m(y)=\sum_{n=0}^{\infty} \int_{E_{n}} \frac{1}{1-\hat{\mu}(y)} d m(y)<\infty .
$$

It follows from (21) that series in (5) converges.

Remark 1. If we put in Theorem 1 all $a_{j}=p$ where $p$ is prime, we obtain Theorem 3 of the article [5] for a random walk defined on the group $H_{p}$.

Thus, we have a sufficient condition for the recurrence of a random walk defined on a subgroup of the group of rational numbers $\mathbb{Q}$, not isomorphic to $\mathbb{Z}$. In the following theorem we obtain a necessary condition for the recurrence of a random walk defined on a subgroup of a group of rational numbers $\mathbb{Q}$.

We need the following well-known property of characteristic functions (see e.g. [3, §2]).

Lemma 1. Let $X$ be a second countable locally compact Abelian group, let $\mu$ be a distribution on $X$. The following conditions are equivalent.

(i) The support of the distribution $\mu$ is not contained in any coset of some subgroup in $X$.

(ii) $\{y \in Y:|\hat{\mu}(y)|=1\}=\{0\}$. 
Theorem 2. Let $X=H_{a}$, where $a=\left(a_{1}, a_{2}, \ldots\right), a_{j}>1$. Let $\mu$ be a distribution on $X$ of the form (44). We suppose that in (44) all $q_{j}>0$. The condition

$$
\sum_{n=1}^{\infty} \frac{a_{n+1}}{a_{1} \ldots a_{n} \sqrt{q_{n}}}=\infty
$$

is necessary for recurrence of a random walk defined by $\mu$ on $X$.

Proof. Let sets $E_{n}$ be such as in Theorem 1. We evaluate from above the sum of seriis (15).

Note that the support of the distribution $\mu$ is not contained in any coset of some subgroup in $X$. Then Lemma 1 implies that

$$
\{y \in Y:|\hat{\mu}(y)|<1\}=\{0\} .
$$

Therefore

$$
\frac{1}{1-\hat{\mu}(y)}=\sum_{k=0}^{\infty} \hat{\mu}^{k}(y), \quad y \neq 0 .
$$

By the Lebesgue-Levi theorem we have

$$
\begin{gathered}
\sum_{n=0}^{\infty} \int_{E_{n}} \frac{1}{1-\hat{\mu}(y)} d m_{Y}(y)=\sum_{n=0}^{\infty} \int_{E_{n}} \sum_{k=0}^{\infty} \hat{\mu}(y)^{k} d m_{Y}(y)= \\
\sum_{n=0}^{\infty} \sum_{k=0}^{\infty} \int_{E_{n}} \hat{\mu}(y)^{k} d m_{Y}(y) .
\end{gathered}
$$

It follows from (77) that $\hat{\mu}(y) \leq q_{0} \cos \frac{\pi}{a_{1}}+q_{1}+q_{2}+\ldots=1-q_{0}\left(1-\cos \frac{\pi}{a_{1}}\right)$ for $y \in E_{0}$. Hence

$$
\int_{E_{0}} \frac{1}{1-\hat{\mu}(y)} d m(y)<\frac{a_{1}-1}{a_{1} q_{0}\left(1-\cos \frac{\pi}{a_{1}}\right)} .
$$

Besides taking into account (13), we note that

$$
\sum_{n=1}^{\infty} \int_{E_{n}} d m(y)=\frac{1}{a_{1}}
$$

Hence

$$
\sum_{n=0}^{\infty} \int_{E_{n}} \sum_{k=0}^{\infty} \hat{\mu}(y)^{k} d m_{Y}(y)=C_{1}+\sum_{n=1}^{\infty} \sum_{k=1}^{\infty} \int_{E_{n}} \hat{\mu}(y)^{k} d m_{Y}(y)
$$

where $C_{1}$ is a constant which does not depend on $n$.

We evaluate from above $\hat{\mu}(y)$ for $y \in E_{n}$. It is obvious that

$$
\begin{gathered}
\hat{\mu}(y)=\sum_{j=0}^{\infty} q_{j} \cos b_{j+1} \leq \sum_{j=0}^{n} q_{j} \cos b_{j+1}+q_{n+1}+q_{n+2}+\ldots \\
=\sum_{j=0}^{n} q_{j} \cos b_{j+1}+1-q_{0}-\ldots-q_{n}=1-\sum_{j=0}^{n} q_{j}\left(1-\cos b_{j+1}\right) .
\end{gathered}
$$


Since $y \in E_{n}$, it follows from (11) that $b_{n+1} \in\left[\frac{2 \pi k}{a_{n+1}}-\frac{\pi}{a_{1} \ldots a_{n+1}}, \frac{2 \pi k}{a_{n+1}}+\frac{\pi}{a_{1} \ldots a_{n+1}}\right](k=$ $\left.1, \ldots, a_{n+1}-1\right)$. Hence for any $n$ the inequality

$$
1-\cos b_{n+1} \geq 1-\cos \left(\frac{2 \pi}{a_{n+1}}-\frac{\pi}{a_{1} \ldots a_{n+1}}\right)
$$

is fulfilled. If $a_{n+1}=2$ or $a_{n+1}=3$ than $\frac{\pi}{2}<\frac{2 \pi}{a_{n+1}}-\frac{\pi}{a_{1} \ldots a_{n+1}}<\pi$. Hence, taking into account (18) and (27), we have

$$
1-\cos b_{n+1} \geq 1>\frac{2}{a_{n+1}^{2}} .
$$

If $a_{n+1}>3$ then $0<\frac{2 \pi}{a_{n+1}}-\frac{\pi}{a_{1} \ldots a_{n+1}}<\frac{\pi}{2}$. Hence, taking into account (27) and (18), we have

$$
1-\cos b_{n+1} \geq \frac{2}{\pi^{2}}\left(\frac{2 \pi}{a_{n+1}}-\frac{\pi}{a_{1} \ldots a_{n+1}}\right)^{2} \geq \frac{2}{a_{n+1}^{2}}
$$

Recall also that $b_{n} \in\left[-\frac{\pi}{a_{1} \ldots a_{n}}, \frac{\pi}{a_{1} \ldots a_{n}}\right]$ for $y \in E_{n}$. Putting $t=b_{n}$ and taking into account (6), we can rewrite estimate (26) in the following way

$\hat{\mu}(y) \leq 1-q_{0}\left(1-\cos a_{2} \ldots a_{n} t\right)-q_{1}\left(1-\cos a_{3} \ldots a_{n} t\right)-\ldots-q_{n-2}\left(1-\cos a_{n} t\right)-q_{n-1}(1-\cos t)-C_{2} \frac{q_{n}}{a_{n+1}^{2}}$

where $t \in\left[-\frac{\pi}{a_{1} \ldots a_{n}}, \frac{\pi}{a_{1} \ldots a_{n}}\right]$.

Put $\tilde{t}=a_{2} \ldots a_{n} t$. Then $\tilde{t} \in\left[-\frac{\pi}{a_{1}}, \frac{\pi}{a_{1}}\right]$. We can rewrite estimate (301) in the following way

$\hat{\mu}(y) \leq 1-q_{0}(1-\cos \tilde{t})-q_{1}\left(1-\cos \frac{\tilde{t}}{a_{2}}\right)-\ldots-q_{n-2}\left(1-\cos \frac{\tilde{t}}{a_{2} \ldots a_{n-1}}\right)-q_{n-1}\left(1-\cos \frac{\tilde{t}}{a_{2} \ldots a_{n}}\right)-C_{2} \frac{q_{n}}{a_{n+1}^{2}}$,

where $\tilde{t} \in\left[-\frac{\pi}{a_{1}}, \frac{\pi}{a_{1}}\right]$.

Taking into account (18) we obtain

$$
1-\cos \frac{\tilde{t}}{a_{2} \ldots a_{j}} \geq \frac{2 \tilde{t}^{2}}{\pi^{2} a_{2}^{2} \ldots a_{j}^{2}}
$$

Thus we can continue inequality (31)

$$
\hat{\mu}(y) \leq 1-\frac{2 \tilde{t}^{2}}{\pi^{2}}\left(q_{0}+\frac{q_{1}}{a_{2}^{2}}+\frac{q_{2}}{a_{2}^{2} a_{3}^{2}}+\ldots+\frac{q_{n-1}}{a_{2}^{2} a_{3}^{2} \ldots a_{n}^{2}}\right)-C_{2} \frac{q_{n}}{a_{n+1}^{2}} \leq e^{-C_{3} \tilde{t}^{2}-C_{4} \frac{q_{n}}{a_{n+1}^{2}}},
$$

where constants $C_{3}$ and $C_{4}$ are positive and do not depend on $n$.

Note that if $y=\left(y_{1}, y_{2}, \ldots\right) \in Y$ then $\tilde{t}=a_{2} \ldots a_{n} \arg y_{n}$. Hence we can rewrite estimate (32) in the folowing way.

$$
\hat{\mu}(y) \leq e^{-C_{3}\left(a_{2} \ldots a_{n} \arg y_{n}\right)^{2}-C_{4} \frac{q_{n}}{a_{n+1}^{2}}}
$$

Fix $n$. The mapping $f: Y \longrightarrow \mathbb{T}$ is defined by the formula $f(y)=\arg y_{n}$. It is easy to see that this mapping transform the Haar measure on $Y$ into the Haar measure on $\mathbb{T}$. We use the 
formula of the change of variable in the integral. Then

$$
\int_{E_{n}} \hat{\mu}^{k}(y) d m_{Y}(y) \leq \int_{E_{n}} e^{-C_{3} k\left(a_{2} \ldots a_{n} \arg y_{n}\right)^{2}-C_{4} k \frac{q_{n}}{a_{n+1}^{2}}} d m_{Y}(y)=\int_{-\pi / a_{1} \ldots a_{n}}^{\pi / a_{1} \ldots a_{n}} e^{-C_{3} k\left(a_{2} \ldots a_{n} t\right)^{2}-C_{4} k \frac{q_{n}}{a_{n+1}^{2}}} d t .
$$

Next, we change variable $s=a_{2} \ldots a_{n} t$. We have

$$
\int_{-\pi / a_{1} \ldots a_{n}}^{\pi / a_{1} \ldots a_{n}} e^{-C_{3} k\left(a_{2} \ldots a_{n} t\right)^{2}-C_{4} k \frac{q_{n}}{a_{n+1}^{2}}} d t=\frac{1}{a_{2} \ldots a_{n}} \int_{-\pi / a_{1}}^{\pi / a_{1}} e^{-C_{3} k s^{2}-C_{4} k \frac{q_{n}}{a_{n+1}^{2}}} d s \leq C_{5} \frac{1}{a_{1} \ldots a_{n}} \frac{e^{-C_{4} k \frac{q_{n}}{a_{n+1}^{2}}}}{\sqrt{k}}
$$

where $C_{5}$ is a constant which does not depend on $n$ and $k$.

Thus

$$
\sum_{n=1}^{\infty} \sum_{k=1}^{\infty} \int_{E_{n}} \hat{\mu}(y)^{k} d m_{Y}(y) \leq C_{5} \sum_{n=1}^{\infty} \frac{1}{a_{1} \ldots a_{n}} \sum_{k=1}^{\infty} \frac{e^{-C_{4} k \frac{q_{n}}{a_{n+1}^{2}}}}{\sqrt{k}}
$$

Since $\sum_{k=1}^{\infty} \frac{e^{-a k}}{\sqrt{k}}=O\left(\frac{1}{\sqrt{a}}\right)$ (see e.g. [5]), we can continue inequality (36) in the following way

$$
\sum_{n=1}^{\infty} \sum_{k=1}^{\infty} \int_{E_{n}} \hat{\mu}(y)^{k} d m_{Y}(y) \leq C_{6} \sum_{n=1}^{\infty} \frac{a_{n+1}}{a_{1} \ldots a_{n} \sqrt{q_{n}}}
$$

where $C_{6}$ is a constant which does not depend on $n$ and $k$.

Arguing in the same way as at the end of proof of Theorem 1, we obtain that condition (26) is necessary for reccurence of a random walk defined by $\mu$.

Remark 2. If we put in Theorem 2 all $a_{j}=p$ where $p$ is prime, we obtain Theorem 4 of the article [5] for a random walk defined on the group $H_{p}$.

\section{References}

[1] Darling, D. A., Erdos, P. On the recurrence of a certain chain, Proc. Amer. Math. Soc. 19 (1968), 336-338.

[2] Dudley, R. M. Random walks on Abelian groups. Proc. Amer. Math. Soc. 13, (1962), $447-450$.

[3] G. M. Feldman, Functional Equations and Characterization Problems on Locally Compact Abelian Groups, EMS Tracts Math. 5, Eur. Math. Soc., Z"urich, 2008.

[4] Flatto, L., Pitt, J. Recurrence criteria for random walks on countable Abelian groups. Illinois J. Math. 18, (1974), 1-19.

[5] Freig, Nabil; Molchanov, S.A. On random walks on Abelian groups with infinite number of generators. (Russian) Vestn. Mosk. Univ., Ser. I 1978, No.5, 22-29 (1978).

[6] E. Hewitt, and K.A. Ross, Abstract Harmonic Analysis, vol. 1, Springer-Verlag, Berlin, Gottingen, Heildelberg, 1963. 
[7] Kasimjanova, M.A. The recurrence of invariant Markov chains on a class of Abelian groups. (Russian) Vestn. Mosk. Univ., Ser. I 1981, No.3, 3-7 (1978).

[8] Kesten, S., Spitzer, F. Random walk on countably infinite Abelian groups. Acta. Math. 114, (1965), 237-265. 